Supporting Information Table 1. Analysis of bioclimatic variable importance for the habitat suitability models of M. gentilis and M. onobrychidis .

\section{Jackknife analysis}

$\%$ contribution Train gain with ${ }^{1} \quad$ Train gain without $^{2} \quad$ Test gain with $^{3} \quad$ Test gain without $^{4}$

M. gentilis Habitat Suitability Model

Mean Temperature of Warmest Quarter

Mean Temperature of Coldest Quarter

Precipitation of Wettest Quarter

Precipitation of Driest Quarter

Precipitation of Warmest Quarter

Temperature Annual Range*

Mean Temperature of Wettest Quarter

Mean Temperature of Driest Quarter

$\begin{array}{ll}0.86 & 2.07 \\ 11.04 & 2.01 \\ 6.99 & 2.00 \\ 21.74 & 1.94 \\ 31.76 & 2.03 \\ 16.18 & 2.02 \\ 2.44 & 2.07 \\ 8.99 & 2.00\end{array}$

M. onobrychidids Habitat Suitability Model

Mean Temperature of Warmest Quarter

Mean Temperature of Coldest Quarter

$\begin{array}{ll}1.4787 & 1.7403 \\ 3.9816 & 1.7378 \\ 4.5882 & 1.6758 \\ 2.3827 & 1.7315 \\ 67.8178 & 1.6965 \\ 2.9892 & 1.7181 \\ 5.2406 & 1.7035 \\ 11.5212 & 1.6723\end{array}$

1.7403

1.7378

Precipitation of Wettest Quarter

Precipitation of Driest Quarter

Precipitation of Warmest Quarter

Temperature Annual Range*

Mean Temperature of Wettest Quarter

Mean Temperature of Driest Quarter
0.05

0.67

0.04

0.81

1.06

0.71

0.61

0.67

0.1674

0.3641

0.4851

0.4892

1.3479

0.2196

0.7823

0.5301

$\begin{array}{ll}2.12 & 0.03 \\ 2.04 & 0.70 \\ 1.99 & 0.02 \\ 2.14 & 0.56 \\ 2.08 & 1.06 \\ 2.08 & 0.76 \\ 2.13 & 0.68 \\ 2.04 & 0.68\end{array}$

\subsection{7}

2.0722

1.969

2.0437

1.987

2.0194

2.0125

1.9397
0.03

0.70

0.02

1.06

0.76

0.68

0.143

0.3622

0.511

0.5273

1.3861

0.2747

0.8024

0.5184

*Max Temperature of Warmest Month - Min Temperature of Coldest Month.

${ }^{1}$ Training gain achieved with each climate variable in isolation.

${ }^{2}$ Training gain achieved excluding each variable in turn.

${ }^{3}$ Test gain achieved with each climate variable in isolation.

${ }^{4}$ Test gain achieved excluding each variable in turn. 